\title{
585.
}

\section{A NEW THEOREM ON THE EQUILIBRIUM OF FOUR FORCES ACTING ON A SOLID BODY.}

[From the Philosophical Magazine, vol. xxxi. (1866), pp. 78, 79; Camb. Phil. Soc. Proc. vol. I. (1866), p. 235.]

DEFINING the "moment of two lines" as the product of the shortest distance of the two lines into the sine of their inclination, then, if four forces acting along the lines 1, 2, 3, 4 respectively are in equilibrium, the lines must, as is known (Möbius), be four generating lines of an hyperboloid; and if 12 denote the moment of the lines 1 and 2 , and similarly 13 the moment of the lines 1 and 3 , \&c., the forces are as

$$
\sqrt{23.34 .42}: \sqrt{34.41 .13}: \sqrt{41.12 .24}: \sqrt{12.23 .31 .}
$$

Calling the four forces $P_{1}, P_{2}, P_{3}, P_{4}$, it follows as a corollary that we have

$$
P_{1} P_{2} \cdot 12=12.34 \sqrt{13.42} \cdot \sqrt{14.23}=P_{3} P_{4} \cdot 34
$$

viz. the product of any two of the forces into the moment of the lines along which they act is equal to the product of the other two forces into the moment of the lines along which they act,-which is equivalent to Chasles's theorem, that, representing a force by a finite line of proportional magnitude, then in whatever way a system of forces is resolved into two forces, the volume of the tetrahedron formed by joining the extremities of the two representative lines is constant.

C. IX. 